\title{
Global gene expression analyses of the alkamide-producing plant Heliopsis longipes supports a polyketide synthase- mediated biosynthesis pathway
}

\author{
Génesis V Buitimea-Cantúa ${ }^{1}$, Nayelli Marsch-Martinez ${ }^{1}$, Patricia Ríos-Chavez ${ }^{2}$, Alfonso Méndez-Bravo ${ }^{3}$, Jorge \\ Molina-Torres ${ }^{\text {Corresp. } 4}$ \\ ${ }^{1}$ Department of Biotecnologia y Bioquímica, CINVESTAV Unidad Irapuato, Irapuato, Guanajuato, Mexico \\ 2 Instituto de Investigaciones Químico-Biológicas, Universidad de San Nicolás de Hidalgo, Morelia, Michoacan, Mexico \\ 3 Laboratorio Nacional de Análisis y Síntesis Ecológica, CONACYT - Escuela Nacional de Estudios Superiores, Morelia, Michoacan, Mexico \\ 4 Department of Biotecnología y Bioquímica, CINVESTAV Unidad Irapuato, Irapuato, Guanajuato, Mexico \\ Corresponding Author: Jorge Molina-Torres \\ Email address: jmolinat@cinvestav.mx
}

Background. Alkamides are plant-specific bioactive molecules. They are low molecular weight Nsubstituted $\alpha$-unsaturated acyl amides that display biological explicit activities in different organisms from bacteria, fungi, insects to mammals and plants. The acyl chain has been proposed to be biosynthesized from a fatty acid, however, this has not been demonstrated yet. Heliopsis longipes (Asteraceae) accumulates in root a C10 alkamide called affinin in its roots, but not in leaves The closely related species Heliopsis annua does not produce alkamides. To elucidate the biosynthetic pathway of the alkamides acyl chain, a comparative global gene expression analysis contrasting roots and leaves of both species was performed.

Methods. Transcriptomics analysis allowed to identify genes highly expressed in $\mathrm{H}$. longipes roots, but not in tissues and species that do not accumulate alkamides. The first domain searched was the Ketosynthase (KS) domain. The phylogenetic analysis using sequences of the KS domain of FAS and PKS from different organisms, revealed that KS domains of the differentially expressed transcripts in $H$. longipes roots and the KS domain found in transcripts of $E$. purpurea, another alkamides producer species, were grouped together with a high bootstrap value of $100 \%$, sharing great similarity. Among the annotated transcripts, we found some coding for the enzymatic domains KS, AT, ACP, DH, OR and TE, which presented higher expression in $H$. longipes roots than in leaves. The expression level of these genes was further evaluated by qRT-PCR. All unigenes tested showed higher expression in $\mathrm{H}$. longipes roots than in any the other samples. Based on this and considering that the acyl chain of affinin presents unsaturated bonds at even $\mathrm{C}$ numbers, we propose a new putative biosynthesis pathway mediated by a four modules polyketide synthase (PKS).

Results. The global gene expression analysis led to the selection of a set of candidate genes involved in the biosynthesis of the acyl chain of affinin, suggesting that it may be performed by a non-iterative, partially reductive, four module type I PKS complex (PKS alk) previously thought to be absent from the plant kingdom. 


\section{Global gene expression analyses of the alkamide-producing plant Heliopsis}

2 longipes supports a polyketide synthase-mediated biosynthesis pathway

3

4 Génesis V. Buitimea-Cantúa ${ }^{1}$, Nayelli Marsch-Martínez², Patricia Ríos Chávez ${ }^{3}$, Alfonso

5 Méndez-Bravo ${ }^{4}$, and Jorge Molina-Torres ${ }^{1 *}$

6

$7 \quad{ }^{1}$ Laboratorio de Fitobioquímica, CINVESTAV Unidad Irapuato, Department of Biotecnología y

8 Bioquímica, Irapuato, Guanajuato, México

9 2Laboratorio de Identidad Celular, CINVESTAV Unidad Irapuato, Department of Biotecnología

10 y Bioquímica, Irapuato, Guanajuato, México

11 Instituto de Investigaciones Químico-Biológicas, Universidad de San Nicolás de Hidalgo,

12 Morelia, Michoacán, México.

23

24

25

26

27
${ }^{4}$ CONACYT - Escuela Nacional de Estudios Superiores, Laboratorio Nacional de Análisis y

Síntesis Ecológica, Universidad Nacional Autónoma de México, Morelia, Michoacán, México.

\section{*Corresponding Author:}

Dr. Jorge Molina-Torres

Km. 9.6 Libramiento Norte, Irapuato, Guanajuato, CP 36824. Mexico.

Email address: jmolinat@cinvestav.mx 


\section{Abstract}

29 Background. Alkamides are plant-specific bioactive molecules. They are low molecular weight

$30 N$-substituted $\alpha$-unsaturated acyl amides that display biological explicit activities in different

31 organisms from bacteria, fungi, insects to mammals and plants. The acyl chain has been

32 proposed to be biosynthesized from a fatty acid, however, this has not been demonstrated yet.

33 Heliopsis longipes (Asteraceae) accumulates a C10 alkamide called affinin in its roots, but not in

34 leaves. The closely related species Heliopsis annua does not produce alkamides. To elucidate the

35 biosynthetic pathway of the alkamides acyl chain, a comparative global gene expression analysis

36 contrasting roots and leaves of both species was performed.

37 Methods. Transcriptomics analysis allowed to identify genes highly expressed in H. longipes roots, but not in tissues and species that do not accumulate alkamides. The first domain searched was the Ketosynthase (KS) domain. The phylogenetic analysis using sequences of the KS domain of FAS and PKS from different organisms, revealed that KS domains of the differentially expressed transcripts in H. longipes roots and the KS domain found in transcripts of Echinaceae purpurea, another alkamides producer plant species, were grouped together with a high bootstrap value of $100 \%$, sharing great similarity. Among the annotated transcripts, we found some coding for the enzymatic domains KS, AT, ACP, DH, OR and TE, which presented higher expression in H. longipes roots than in leaves. The expression level of these genes was further evaluated by RT-qPCR. All unigenes tested showed higher expression in H. longipes roots than in any the other samples. Based on this and considering that the acyl chain of affinin presents unsaturated bonds at even $\mathrm{C}$ numbers, we propose a new putative biosynthesis pathway mediated by a four modules polyketide synthase (PKS). non-iterative, partially reductive, four module type I PKS complex (PKS alk), previously thought to be absent from the plant kingdom. 


\section{Introduction}

58 Alkamides are plant natural products that display diverse biological activities in different phyla,

59 such as antifungal, antibacterial, antimalarial, insecticidal, quorum sensing blockage, anaesthetic, 60 analgesic and pungent activities (Barbosa et al., 2016). They also contribute to plant growth

61 promotion and gene defence induction and interact with intercellular signal molecules in

62 humans, bacteria and plants (Ramírez-Chávez et al., 2004; Méndez-Bravo et al., 2011).

63 Chemically, alkamides are low molecular weight, $<400 \mathrm{Da}$, N-substituted $\alpha$-unsaturated acyl

64 amides. They consist of a straight $\alpha$-unsaturated C8 to C18 acyl chain condensed to different

65 amines. The most abundant alkamide acyl chain reported is C10 (Greger, 2016). The acyl chain

66 of these rather simple structures presents unsaturated double bonds found isolated $(E)$ or

67 conjugated ( $E, E$ or $E, Z$ ), either in the $\alpha$-, intermediate or methyl- $\omega$ position, starting at an even

$68 \mathrm{C}$ position. Alkamides are plant metabolites comprising a group close to 300 structures produced

69 by a limited number of plant species belonging taxonomically to only eight families. A database

70 of alkamides and other acyl-amides has been recently compiled (Boonen et al., 2012).

71 Alkamides are synthetized by plants, and similar, higher molecular weight $\gtrsim 800$ Da, onnamide-

72 type polyketides are produced by sponge from symbiotic bacteria (Piel 2009). In plants,

73 alkamide accumulation is restricted to specific tissues such as roots, flowers, fruits or seeds,

74 except in the genus Acmella (Asteraceae) where alkamides have been detected in the whole

75 plant. Several species containing high levels of alkamides are found in the Asteraceae,

76 Piperaceae and Rutaceae; being more frequent in the Asteraceae family (Christensen \& Lam

77 1991; Parmar et al., 1997; Yang, 2008). The Heliopsis genus, which belongs to the Heliantheae

78 tribe in the latter family, contains 16 species, most of them endemic to Mesoamerica (García-

79 Chávez, Ramírez-Chávez \& Molina-Torres 2004; Ramírez-Noya, González-Elizondo \& Molina-

80 Torres, 2011). Plants of this genus produce over 30 different alkamides. Their acyl chain length

81 varies from $\mathrm{C} 10$ to $\mathrm{C} 18$, with the amine moiety being usually either isobutylamide or 2-

82 methylbutylamide. The most commonly reported functional groups of plant alkamides are

83 isobutylamine as the amine moiety and the C10 acyl chain. The $\alpha$-double bond of the acyl

84 moiety is the main characteristic of alkamides. In several genus e.g. Heliopsis, other double

85 bonds vary in position but are frequently conjugated at positions 4, 6, 8, 10 or 14. Triple

86 (acetylenic) bonds, when present, are in a position corresponding to that of the double bonds, 
87 most frequently occurring as acetylenic or methyl acetylenic in the $\omega$-position. When more than 88 one acetylenic bond is present, these are conjugated. In those alkamides where the acetylenic 89 bond is at the $\omega$-position, the acyl chain presents an odd number of carbons. In Heliopsis 90 longipes, alkamides accumulate in the roots but are not present in the vegetative aerial tissue. In 91 contrast, alkamides have not been detected in leaves or roots of the related species Heliopsis 92 апnиа. The most abundant alkamide in $H$. longipes roots is $N$-isobutyl-2E,6Z,8E-decatrienamide, 93 named affinin. It represents over $90 \%$ of the total alkamide content in well-developed roots. Other $\mathrm{C} 10$ acyl chain alkamides in this tissue are structurally related to affinin, such as the $\mathrm{N}-2$ methylbutyl-2E,6Z,8E-decatrienamide and the bornyl ester of C10-2E,6Z,8E (Fig. 1). They contain the same acyl chain, $\mathrm{C} 10-2 E, 6 Z, 8 E$, and therefore these alkamides might be produced in a similar biosynthetic pathway not yet completely elucidated. Information is available regarding the biosynthetic pathway of the amine moiety. Studies performed on some species of the Asteraceae have suggested that the amine moiety isobutyl of affinin is derived from an amino acid that is first decarboxylated and then linked through an amide bond to the acyl chain. This was confirmed through feeding experiments using L- $\left[{ }^{2} \mathrm{H} 8\right]$-valine, employing Acmella radicans as a model (Cortez-Espinosa et al., 2011). Moreover, a recent detailed study on alkamides biosynthesis in several Echinacea species using transcriptomics, metabolite profiling and isotopic labelling, validated that a branched-chain amine is acylated with the fully extended acyl chain. A pyridoxal phosphate-dependent decarboxylase was identified as the enzyme responsible for generating the amide alkamide moiety (Rizhsky et al., 2016).

The acyl chain of alkamide molecules has been considered to arise from a polyunsaturated fatty acid. This alkamide biosynthesis pathway mediated by fatty acids was proposed several decades ago based on feeding experiments with ${ }^{14} \mathrm{C}$ - and ${ }^{3} \mathrm{H}$-labelled acetate precursors, demonstrating that these derived from acetate. More recently a proposed path suggested that the alkamide acyl chain was mediated by oleic acid (C18:1) by successive $\beta$-oxidation, dehydration, and isomerisation (Greger, 1984; Minto \& Blacklock, 2008). However, this biosynthetic pathway for alkamides has not been experimentally confirmed. Fatty acids are distributed among all living organisms, indicating that all organisms possess a functional Fatty Acid Synthase (FAS) pathway, while alkamides are only present in some plant species. Structurally the double bonds of the acyl chain of alkamides are clearly different from those of fatty acids and their derivatives, which are always in the cis configuration $(Z)$ and non-conjugated. The double bonds of fatty 
118 acids are incorporated by desaturases at a site different from that on which the acyl chain is 119 synthesised and are strictly positioned in relation to the first desaturation $9 Z$, such as $12 Z, 15 Z$, or $12018 Z$ (Brown, Slabas \& Rafferty, 2009). In contrast, the acyl chains of alkamides contain double 121 bonds in different positions of the chain. These double bonds are largely present in the trans 122 configuration $(E)$ but may be in cis $(Z)$ when these are conjugated, in contrast to those found in 123 fatty acids. These facts, together, suggest that the biosynthesis of the acyl chain of alkamides 124 could occur through an alternative pathway. Biochemical or genetic information to support the 125 fatty acid or any alternative biosynthetic pathway of alkamides is still scarce, essentially because 126 alkamides are produced in non-model plants for which the genomes have not yet been 127 sequenced. Whole genome sequencing has been successfully used to elucidate biosynthetic pathways in microorganisms, such as bacteria. However, plant genomes are much larger than bacterial genomes, which increases the cost and time associated with sequencing, and plant genomes exhibit particularities such as alternative splicing that can give rise to different transcript versions of a single gene. Therefore, transcript profiling using high-throughput RNA sequencing (RNA-Seq) is a first-rate alternative to investigate the biosynthetic pathways of metabolites in plants without sequenced genomes (Osbourn \& Lanzotti, 2009). Transcriptomic or global expression analyses can be a powerful tool, and comparison of cell or tissue transcriptomes that do or do not produce a metabolite of interest, can allow the discovery of the genes involved in specific biosynthetic pathways. Transcriptome sequencing by RNA-Seq has been successfully used to profile the expression patterns from different plant tissues, facilitating the acquisition of additional knowledge on secondary metabolite biosynthesis in plants, especially from non-model plant species (Osbourn \& Lanzotti, 2009).

In this study we performed global expression analysis by RNA-Seq and compared the transcriptomes of tissues and species that accumulate or do not accumulate alkamides.

Differentially expressed candidate genes were identified and based on their nature, an alternative putative biosynthesis pathway for the acyl chain of alkamides is proposed. The results here obtained provide a robust basis for subsequent informed experiments to confirm and further understand the proposed biosynthesis pathway of alkamides, specifically for the acyl chain of these molecules.

\section{Materials \& Methods}


148 Biological material. Heliopsis longipes was collected in February 2014 at Puerto de Tablas, 149 Xichú municipality, Sierra Gorda, state of Guanajuato, México (Lat. 211'ㄹ" $20^{\prime \prime}$, Long. $150100^{\circ} 05^{\prime} 19^{\prime \prime} \mathrm{W}$, Alt. 2,589 m above sea level). Voucher specimens were deposited at Instituto de 151 Ecología, Centro Regional del Bajío. Herbarium IEB (Voucher 263787). Heliopsis annua was 152 collected in October 2014 in the municipality of Vicente Guerrero, state of Durango, México 153 (Lat. 234' $41^{\prime \prime} \mathrm{N}$, Long. 10400'02" W, Alt. 1,950 m above sea level). Voucher specimens were 154 deposited at CIIDIR Durango Herbarium (Voucher id. D. Ramírez 5543). Fresh roots and leaves 155 of both species were collected separately, washed with distilled water, immediately frozen in 156 liquid nitrogen, and stored at $-80^{\circ} \mathrm{C}$ until processed for RNA extraction.

157 RNA extraction and transcriptome sequencing. Total RNA was extracted from roots and 158 leaves of eight individual plants of each Heliopsis species and processed separately as two 159 replicates, following the protocol established by the PureLinkTM Micro-to-Midi Total RNA 160 Purification System (Invitrogen, Carlsbad, CA, USA). The quality of the RNA samples was 161 evaluated using the RNA 6000 Nanochip on the BioAnalyzer 2100 (Agilent Technologies, Cedar 162 Creek, TX, USA). Complementary DNA libraries were constructed with insert sizes from 339 to 163697 base-pairs (bp) and sequenced using the Illumina HiSeq 2500 platform (Illumina, San Diego, 164 CA, USA) with paired-end reads of $100 \mathrm{bp}$, at the Unidad de Servicios Genómicos of 165 CINVESTAV, Irapuato, Guanajuato, México.

166

167

168

169

170

171

172

173

174 175 2011). 
176 Gene function annotation. The consensus contig sequence files were annotated against the non177 redundant protein (NR) database from the National Center for Biotechnology Information 178 (NCBI; www.ncbi.nlm.nih.gov), Protein Family (PFAM; https://pfam.xfam.org) database, which contains a large collection of protein families, each represented by multiple sequence alignments and hidden Markov models, and Swiss-Prot database (https://www.uniprot.org/), with an e-value cut-off of 1e-5. Gene Ontology (GO) analyses were further used to categorize the function of the unigenes with Blast2GO 5 (https://www.blast2go.com/). Using the basic local alignment search tool (BLAST), the Arabidopsis thaliana transcription factor (TF) database was queried to identify TFs among all unigenes (identity N80\%) (Guo et al., 2005).

Differential expression analysis. To estimate the expression level of each transcript in the different tissue samples, high-quality reads from each sample were mapped onto the final transcriptome assembly using the RSEM software (Li \& Dewey, 2011). Read counts were normalized by calculating fragments per kilobase of exon model per million mapped reads (FPKM) values. Differential gene expression analysis was performed using the EdgeR (Bioconductor, R) software (Robinson, McCarthy \& Smyth, 2010). A p-value cut-off $\leq 0.05$ [multiple test correction: false discovery rate (FDR)] and $\log 2$ fold change (FC) $\geq 2$ were used to identify significant differences in transcript expression.

Phylogenetic analysis of KS domains. The amino acid sequences of the KS domains of $H$. longipes and $H$. annua were obtained from the translated assembled RNA-Seq data. The KS domains were corroborated by BLAST (https://blast.ncbi.nlm.nih.gov/Blast.cgi) and PFAM, a database of large collection with protein families (https://pfam.xfam.org). The domains compared were: the KS domains identified in H. annua roots (A1-A4); the KS domains upregulated in H. longipes roots (B1-B4); the KS domains related to FAS from H. annua (C1C11); the KS related to FAS from $H$. longipes (D1-D11); the KS of the type III PKS from $H$. апnиa (E1-E4); and the KS of the type III PKS from H. longipes (F1-F4). These KS sequences were compared with KS domains of several microorganisms, plant and mammal species obtained from the NCBI database (www.ncbi.nlm.nih.gov) (Table S1). The KS sequence of Echinacea purpurea was retrieved from the medicinal plant database of the Plant Biology Department, Michigan State University, East Lansing, MI, USA (http://medicinalplantgenomics.msu.edu/). Echinacea purpurea also belongs to tribe Heliantheae within the Asteraceae and produces 
206 alkamides that are structurally related to affinin (Rizhsky et al., 2016). After selection, sequences 207 were aligned using ClustalW with the default parameters (Chenna, 2003). Phylogenetic and 208 molecular evolutionary analyses were conducted in MEGA 7 using the maximum likelihood 209 method based on the JTT matrix-based model and 1,000 bootstrap replications (Jones et al., 210 1992; Kumar, Stecher \& Tamura, 2016).

211 Quantitative RT-PCR. Reverse Transcriptase quantitative polymerase chain reaction assays 212 (RT-qPCR) were performed using the Applied Biosystems 7500 Fast Real-Time PCR system 213 (Applied Biosystems, Foster City, CA, USA) with the Brilliant II SYBR Green RT-qPCR 1-Step 214 Master Mix Kit (Agilent Technologies, Cedar Creek, TX, USA). Gene-specific primers for the 215 selected genes in the acyl chain biosynthesis pathway were designed using Primer3 216 (www.primer3.org) and are shown in Table S2. The RT-qPCR for each gene was performed 217 using three biological and three technical replicates. Actin served as the internal control for 218 comparison. Relative transcript expression levels were calculated using the $2^{-\Delta \Delta \mathrm{CT}}$ method 219 (Chenna, 2003; Livak \& Schmittgen, 2001; Bustin et al., 2009). Differential gene expression 220 analysis based on the RT-qPCR results was performed for $H$. longipes roots vs. leaves and $H$. 221 longipes roots vs. H. annua roots.

\section{Results}

223

224

225

226

227

228

229

230

231

232

233

234

RNA sequencing and de novo transcriptome assembly. To find differentially expressed genes that could encode enzymes involved in alkamide biosynthesis, transcriptomes of producing and non-producing tissues and species were obtained. Roots of $H$. longipes produce alkamides, while leaves do not. Neither roots nor leaves of H. annua produce these compounds. RNA was isolated from each tissue and RNA-Seq was performed. Each library was sequenced using the Illumina Hiseq2500 platform. Adapter sequences, low quality reads, and reads that were shorter than 50 bp were removed, yielding more than 275,538,680 clean reads in the $2 \times 100$ paired-end formats (c. $27.7 \mathrm{Gbp}$ ). Roots and leaves of $H$. longipes generated 45,622,318 and 31,731,475 raw reads, respectively, whereas roots and leaves of $H$. annua generated 30,326,119 and 30,089,428 raw reads, respectively (Table 1A). Clean reads were assembled de novo using the Trinity package, developed specifically for next-generation short-read sequences (Garber et al., 2011). Based on the high-quality reads, 165,770 unigenes in $H$. longipes and 155,457 unigenes in H. annua were 
235 assembled (Table 1B). In terms of size distribution: 70\% of the H. longipes unigenes and $77 \%$ of 236 the H. annua unigenes were between 200 and 1,000 bp. Transcripts in H. longipes had a 237 maximum size of 14,461 bp, an average size of $906 \mathrm{bp}$, and a minimum size of $200 \mathrm{bp}$. In $H$. 238 annua, transcripts had a maximum size of $15,574 \mathrm{bp}$, an average size of $695 \mathrm{bp}$, and a minimum 239 size of $200 \mathrm{bp}$.

240 Functional annotation. Functional annotations of the transcriptomes revealed functional 241 predictions for 81,682 transcripts for $H$. longipes and 68,166 transcripts for $H$. annua. The 242 sequences that did not present a significant hit with a known protein were most likely short 243 sequences with less than $200 \mathrm{bp}$, and probably represented genes that have not yet been 244 functionally characterized, untranslated regions or specific genes for Heliopsis species. Based on 245 the top-hit transcript homologies against the NR database, ca. 90\% of the unigenes in both 246 species were highly homologous to proteins from land plants. The Gene Ontology classification 247 system defines concepts/classes used to describe gene function and relationships between these 248 concepts (Ashburner et al., 2000). Using this system, we were able to assign 29,593 GO terms to 249 the unigenes. Annotations were distributed into the three major categories of GO: Molecular 250 Function, molecular activities of gene products; Cellular Component, where gene products are 251 active; and Biological Process, pathways and larger processes comprising the activities of 252 multiple gene products. GO annotations included: for H. longipes 10,796 molecular functions, 253 14,601 biological processes, and 4,153 cellular components; for H. annua 11,018 molecular 254 functions, 14,367 biological processes and 4,251 cellular components (Fig. 2). The distribution 255 of unigenes into the three different GO categories is an indicator of the wide diversity of genes 256 that were present in the roots and leaves transcriptomes of these species. The most commonly 257 assigned functional categories in each species, "catalytic activity" for the molecular function 258 category, and "metabolic process" for the biological process category, were consistent with the 259 results of other non-model plant species producing specialized metabolites (Xiao et al., 2013). As 260 in transcriptomes of other plants, some transcripts encoded proteins of unknown function.

261 TFs play key regulatory roles in the secondary metabolism of plants. The identification, 262 annotation, and classification of TFs in the roots and leaves transcriptomes of $H$. longipes and $H$. 263 annua revealed high homology with 42 known TF families. Among the 1,006 unigenes identified 264 as TFs in H. longipes, the most abundant presented high homology to members of the WRKY 
265 family (241 unigenes), followed by the MYB family (223 unigenes), and members of the GRAS 266 family (80 unigenes). In $H$. annua, among the 938 unigenes related to TFs, the most abundant 267 presented high homology to members of the LSD family (218 unigenes), followed by the WRKY 268 family (162 unigenes), and the members of the HB family (68 unigenes). Finding MYB family 269 members in H. longipes was interesting, since the MYB TFs include a conserved MYB DNA270 binding domain that commonly binds to and regulates genes that code for enzymes involved in 271 metabolic pathways (Ambawat et al., 2013).

272 Differential gene expression analysis. The comparison of transcriptomes obtained from roots 273 and leaves of $H$. longipes and $H$. annua revealed differences in transcript expression among the 274 two tissues and Heliopsis species. Among the 5,818 differentially expressed transcripts identified 275 in $H$. annua roots, compared to H. longipes roots, 4,997 transcripts were down-regulated and 821 276 were up-regulated. The comparison between transcriptomes of H. longipes roots and leaves 277 revealed 2,058 differentially expressed transcripts: 1,399 were down-regulated and 659 were up278 regulated in roots. For both Heliopsis species, the up-regulated transcripts in roots, when 279 compared to their corresponding leaves, encoded some proteins of unknown function, whereas 280 the down-regulated transcripts included proteins related to photosynthesis, carbohydrate 281 metabolism, and other processes. The differential expression analyses also identified 71 genes related to the FAS complex in the two Heliopsis species studied. These genes were highly 283 expressed in $H$. annua leaves. The differential expression analysis highlighted genes encoding 284 enzymes that could be responsible for the biosynthesis of the acyl chain in the structure of alkamides, as represented by the $\mathrm{C} 10-2 E, 6 Z, 8 E$ acyl chain of affinin in $H$. longipes. When 286 comparing the transcriptomes from both species with differential alkamide production and accumulation, we identified a set of genes whose expression was higher in $H$. longipes roots, representing the species and tissue that accumulated the metabolites of interest in the present 289 study (Fig. 3A).

In order to confirm the differential expression of candidate genes in H. longipes and H. annua leaves and roots an analysis by RT-qPCR was performed. Transcripts encoding enzymatic 292 domains that could be involved in the biosynthesis of a molecule with the characteristics of 293 alkamides were sought, choosing those involved in the metabolism of acetate. As such candidate 294 genes encoding the different domains: ketosynthase (KS), acyl transferase (AT), acyl carrier 
295 protein (ACP), oxide reductase (OR), dehydratase (DH) and thioesterase (TE) were selected 296 (Table S2). Actin (ACT), coding a cytoskeleton structural protein, was used as a reference gene

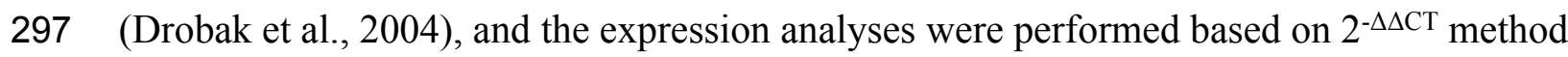
298 (Chenna, 2003). The RT-qPCR data confirmed that the candidate genes were expressed, and that 299 they showed differential expression, being highly expressed in H. longipes roots in comparison 300 to H. longipes leaves and H. апnua leaves and roots (Fig. 3B). RNA-Seq data revealed a good 301 concordance with RT-qPCR expression quantifications. This has also been reported by other 302 authors (Wing et al., 2016; Zhang and Deyholos 2016). Our results also showed that all 303 expression comparisons of the RT-qPCR assays were in fairly good match with the RNA-Seq 304 data, even if the fold-change of some genes in their expression level detected by RNA305 Sequencing and RT-qPCR did not match perfectly. These data confirmed the reliability of the 306 RNA-Seq results. Briefly, as shown in Figure 3 the RNA-Seq and RT-qPCR display consistent expression patterns for the examined genes. Specifically, the expression levels of all unigenes were higher in H. longipes roots than in other plant tissues, which is consistent with the RNASeq data. This indicates that the experimental results are reliable. Therefore, we conclude that RNA-Seq data presented here accurately represent differences in transcript expression between the alkamide-producer tissues (H. longipes roots) and the tissues that do non-produce these compounds (H. longipes leaves, H. annua leaves, and H. annua roots).

Phylogenetic analysis of the Ketosynthase domains among differentially expressed genes. In order to find genes that could be related to the biosynthesis of alkamides, the annotated transcriptomes were used to search for transcripts that coded for conserved domains of fatty acid synthases, but that also were shared with other related enzymes. We noticed that, among the list

317 of differentially expressed transcripts, there were four annotated as coding for proteins

318 containing the KS domain. This domain was chosen for further analyses not only because it is 319 the most conserved domain in the FASs, but it is also present in other related enzymes like the 320 PKS multi-enzymatic complexes and used for phylogenetic studies (You et al., 2014; Gallo, 321 Ferrara \& Perrone, 2013; Kohli et al., 2016). The KS domain catalyzes the condensation of two 322 substrates depending on the type of enzyme, that will form a growing acyl chain (Brown, Slabas $323 \&$ Rafferty, 2009). We expected that enzymes containing this domain would participate in the 324 biosynthesis of the acyl chain of alkamides. The phylogenetic analyses of eukaryotic FASs and PKSs using these conserved KS domains, has been used to emphasize the singularity of the 
326 enzymatic complexes, their evolutionary relationships, taxonomic distribution, and the level of

327

328

329

330

331

332

333

334

335

336

337

338

339

340

341

342

343

344

345

346

347

348

349

350

351

352

353

354

355 conservation of these multi-modular enzymes (You et al., 2014; Gallo, Ferrara \& Perrone, 2013; Kohli et al., 2016).

The list of annotated transcripts differentially and not differentially expressed in $H$. longipes and H. аппиа tissues was screened for transcripts coding proteins containing a KS domain. In order to better identify the type of KS domains coded by the different transcripts, we used the translated transcript sequences to perform a molecular phylogenetic analysis using the maximum likelihood method. The analysis included the KS domains of transcripts upregulated in $H$. longipes roots. As a comparison, also was included KS domains from $H$. longipes leaves and $H$. annua roots and leaves, which did not show differential expression but for which the annotation indicated that they coded proteins related to FAS or PKS type III of plants. These different KS domains were classified as: A, C, D, E or F for transcripts from H. аnnua (roots and leaves) and H. longipes (leaves), that were annotated as coding for a KS domain or KS domains related to FAS or type III PKS; and B for H. longipes roots upregulated transcripts. Other KS domains of FAS and PKS from mammals, microorganisms and other plants, obtained from NCBI listed in Table S1. KS domains encoded in transcripts of Echinacea purpurea were also included.

The results of the maximum likelihood phylogenetic analysis based on $89 \mathrm{KS}$ sequences are presented in Fig. 4. As expected, the KS domains that were annotated as related to FAS from $H$. longipes D1-D11 and H. апnиa C1-C11, are grouped in a clade together with the FAS from Arabidopsis thaliana KS with a high bootstrap value (98\%). This result suggests that these transcripts, obtained from $H$. longipes and $H$. апnиa, encode enzymes with function similar to the KS domain of $A$. thaliana, which belongs to $\beta$-ketoacyl-[acyl-carrier-protein] synthases III (EC 2.3.1.180), enzymes that catalyze the chemical reaction of acetyl-CoA + malonyl-[acyl carrier protein] and produces acetoacetyl-[acyl carrier protein] $+\mathrm{CoA}+\mathrm{CO}_{2}$. These enzymes belong to the family of transferases, and particularly to those transferring groups other than aminoacyl groups. They participate in fatty acid biosynthesis and are found in the dissociated or type II FAS biosynthesis system that occurs in plants and bacteria. The KS domains related to type III PKS are represented by the domain found in chalcone synthases (CHS), the enzymatic complexes mediating the synthesis of flavonoids in plants (Abe \& Morita, 2010). The nondifferentially expressed KS domain sequences that were chosen due to their annotation as type 
356 III PKS from H. longipes (F1-F4) are grouped together with a high bootstrap value of 100\%.

357 However, they are grouped in a clade far apart from the KS of type III PKS from H. annua E1-

358 E4, which cluster in a clade together with the KS of type III PKS from Arabidopsis thaliana with

359 a high bootstrap value of 98\%. Therefore, these transcripts labelled as E1-E4 may share a similar

360 function to the type III from $A$. thaliana. In addition, the translated transcripts of $H$. annua A1-

361 A4 are close to the clade of H. аппиа E1-E4, translated transcripts coding for type III PKS KS

362 domains or Chalcone synthase (CHS). As for the FAS-related sequences, the transcripts coding

363 for these domains are present in both species, which could suggest that they may not be involved

364 in the biosynthesis of the acyl chain of alkamides.

365 The KS domains coded by the transcripts B1-B4 found to be upregulated in H. longipes roots,

366 the species and tissue that produces alkamides, grouped with a high bootstrap value of $100 \%$

367

368

369

370

371

372

373

374

375

376

377

378

379

380

381

382

383

384

385 with KS (3-oxoacyl-[acyl-carrier-protein] synthase) of Echinacea purpurea roots. This group is exclusively formed by translated transcripts of E. purpurea and upregulated in $H$. longipes roots. This association suggest that the KS domains in both species probably have the same function and could be related to alkamide acyl chain biosynthesis, due to their functional nature. Close to this KS clade, KS domains from other plant species include Helianthus annus and Cynara cardunculus. This clade is not far from KS domains from the Chlamydiae phylum microorganism PKS specifically with the two orders: Chlamydiales and Parachlamydiales; and a single class Chlamydiales. These are obligate intracellular Gram-negative bacteria (Gupta et al., 2015).

In summary, the phylogenetic analysis revealed that the KS domains found in the annotated transcriptomes of the two species of the Heliopsis genus under study, are separated into at least four categories associated with plants: 1) The first KS domain, associated with plant FAS, includes general $H$. longipes, $H$. annua, and other plant transcripts; 2) A second domain type found exclusively in $H$. longipes root upregulated transcripts and E. purpurea. This KS domain, present in both species, is possibly related to the biosynthesis of the acyl chain of alkamides in H. longipes and E. purpurea. Interestingly, they were grouped in a clade far from FAS and PKS III KS reported in plants, but closer to microorganism PKS (Hertweck, 2009; Piel, 2010); 3) The third domain type present exclusively in H. longipes, associated with type III PKS is located far from the other KS domain of $H$. longipes, related to the alkamides biosynthesis. These $H$. 
386

387

388

389

390

391

392

393

394

395

396

397

398

399

400

401

402

403

404

405

406

407

408

409

410

411

412

413

414

415

longipes type III KS domain could be associated with other metabolites from H. longipes; 4) The fourth includes two clades of $H$. annua KS domains related to type III PKS.

Proposed model of a pathway for the biosynthesis of the acyl chain of alkamides. The acyl chain of affinin is a straight chain with an even number of carbons, which suggests that acetate units are involved in its biosynthesis as mediated by a FAS. The presence of double bonds in the chain at even positions may be justified by the deficiency of enoyl reductase (ER) enzymes in a modular manner. As observed in polyketides biosynthesis from bacteria, fungi and symbiotic marine microorganisms such as in sponges (Piel, 2009), where unsaturated bonds that are incorporated during the chain elongation. The differentially expressed genes encoding polyketide synthase domains and among the candidate transcripts, four transcripts encoding the AT, KS and $\mathrm{DH}$, seven transcripts encoding the OR and eleven transcripts encoding the ACP enzymatic domains were found. Thus, a PKS pathway using all these enzymatic domains for the biosynthesis of the acyl chain moiety of $H$. longipes alkamides is proposed. The available information about the different domain activities in published PKS systems, allowed the assembly of a model of a biosynthetic pathway that could produce the $C 10-2 E, 6 Z, 8 E$ acyl chain of affinin. This model incorporates the KS, AT, ACP, OR (including the keto reductase (KR) and ER), DH and TE domains previously found. The model proposes a four-module PKS system where the starting precursor molecule is acyl-CoA, and the extender unit is malonyl-ACP (Fig.5).

The proposed sequence of acyl chain biosynthesis, as evidenced by the differential expression supporting a four-modules multi-enzymatic complex, is as follows: in the first module (Aff-1), the AT (GO:0016740) domain accepts and loads the extender unit onto the ACP (GO:0016740) and produces malonyl-ACP. Then, the KS (GO:0016740) domain catalyses a decarboxylative Claisen condensation with the acyl-CoA and the resulting product is a four carbon $(\mathrm{C} 4)$ acyl chain with $\alpha$ - and $\beta$-keto groups. The KR, including OR (GO:0016491) and DH (GO:0016740) domains, sequentially reduce the $\beta$-keto group to a $\beta$-hydroxyl group and an $\alpha$ - $E$ double bond, respectively. The $\alpha$-unsaturated acyl chain is transferred by the AT (GO:0016740) to the second module (Aff-2) and is elongated by the ACP (GO:0016740) with another malonyl-ACP producing a C6-acyl chain. The same enzymatic reactions on the $\beta$-keto group as in the first module occur in module 2 . The product is a C6-2Z,4E-dien-acyl chain, which is transferred to 
416 the module 3 (Aff-3) and condensed with malonyl-ACP to produce a C8-acyl chain with $\alpha$ - and $417 \beta$-keto groups. In this module, which consist of includes the KR including OR (GO:0016491) 418 and DH (GO:0016740) domains, the $\beta$-keto group is reduced to a $\beta$-hydroxyl group and 419 dehydrated to an $\alpha-E$ double bond. The ER (GO:0016491) enzymatic domain, present only in 420 this module, reduces the double bond. In module 4 (Aff-4), the acyl chain C8-4Z,6E is once more 421 condensed with malonyl-ACP to produce an C10-acyl chain with two keto $\alpha$ - and $\beta$ - groups. A $422 \mathrm{KR}$, including OR (GO:0016491) and DH (GO:0016740) domains, in this module reduces the $\beta$ 423 keto group to a $\beta$-hydroxyl group, and the $\mathrm{DH}$ domains to an $\alpha-E$ double bond. The absence of an 424 ER domain in this last module 4 results in the transference of the final $\mathrm{C} 10-2 E, 6 Z, 8 E$-chain to 425 the TE (GO:0016744) domain present at the end of this four module PKS. Table 3S includes the 426 sequence and annotation of unigenes participating in the putative biosynthesis pathway and 427 information about the alignments to Arabidopsis and other plant species.

428 The acyl chain length of the alkamides would depend on the number of modules present in the 429 multi-enzymatic PKS complex, while the position and number of double bonds, would depend 430 on the presence and activity of ER domains following the KR and DH domains, as reported for 431 other PKS complexes (Hertweck, 2009). The combination of all these factors may then lead to 432 the biosynthesis of acyl chains with different structures, which could explain the structural 433 diversity of natural alkamides.

\section{Discussion}

435 The aim of this study was to obtain experimental information on global gene expression in roots 436 and aerial tissues of two Heliopsis species and use this information to identify candidate genes 437 involved in the biosynthesis of the acyl chain of alkamides, based on the fact that alkamides are 438 differentially synthesized in some specific Heliopsis species and tissues. Alkamides accumulate 439 in H. longipes roots, as observed in older plants contain higher levels of alkamides than roots of 440 younger plants (García-Chávez, Ramírez-Chávez \& Molina-Torres, 2004). These compounds are 441 continuously being biosynthesized during plant growth, independently of external factors, 442 suggesting that their biosynthetic genes are constitutively expressed in the tissues where 443 alkamides are produced. RNA-Seq studies have facilitated the discovery of biosynthetic genes 444 responsible for the production of plant-specialized metabolites in non-model plants, through the 
445 sequencing and comparison of transcriptomes of different tissues (Xiao et al., 2013). In the 446 present work, the Illumina HiSeqTM 2500 technology was used to obtain the transcriptomes of 447 roots and leaves of two Heliopsis species, where over $90 \%$ of the assembled unigenes matched 448 with the genomic database of other plants. To the extent of our knowledge, this is the first report 449 about global gene expression analyses of roots and leaves of Asteraceae species. The diversity of 450 the GO terms related to the assembled unigenes, as demonstrated by functional GO assignments, 451 revealed a variety of unigenes involved in specialized metabolite biosynthesis pathways.

452 Differential gene expression analysis allowed to identify genes that were highly expressed in $H$. 453 longipes roots, but not in tissues that do not accumulate these metabolites: $H$. longipes leaves or 454 H. аnnua roots and leaves. Because of their expression pattern, they are very likely related to the 455 biosynthesis of alkamides. Among the annotated transcripts, we found some coding for the 456 enzymatic domains KS, AT, ACP, DH, OR and TE, which presented higher expression in $H$. 457 longipes roots than in leaves (Fig. 3A). The expression level of these genes was further evaluated 458 by RT-qPCR and all unigenes tested showed higher expression in H. longipes roots (Fig. 3B). 459 Given their preferential expression, which could explain why alkamides are produced and 460 accumulate in $H$. longipes roots but not in $H$. longipes leaves nor $H$. annua tissues, these genes

461 462

463 464 465 466 467 468 469 470 471 472 473 of the differentially expressed transcripts in H. longipes roots and the KS domain found in 474 transcripts of E. purpurea, were grouped together with a high bootstrap value of $100 \%$, are potential candidates to code for the enzymes that perform the biosynthesis of the acyl chain moiety of alkamides.

To better understand the biosynthesis of the acyl chain of alkamides and considering the nature of the chemical reactions that could produce such a chain structure, we sought enzymatic domains in the translated differentially expressed transcripts that could perform such reactions. The first domain searched was the KS domain. This domain is very conserved in FAS, and as in other related enzymes, such as the fatty acid elongases (FAE) that act at the end of the FAS steps, or complexes such as PKS. It is responsible for the condensation of two substrates to generate or elongate an acyl chain, depending on where the enzyme that contains it is present. It can be assumed that such a domain would be participating in the initial steps of the biosynthesis of the acyl chain of alkamides. Indeed, transcripts encoding the KS domain were found among

72 the differentially expressed set. The phylogenetic analysis (Fig. 4) revealed that the KS domains 
475 suggesting that they share a common ancestor. This clade that included the KS domain of plants 476 and tissues that produce alkamides can be called "Alkamides clade". The KS domains in the 477 alkamide clade are separated from the KS domains of FAS from H. longipes and H. annua 478 leaves, as well as from the KS of the type III PKS from H. longipes and H. annua. Type III PKS 479 is the chalcone synthase complex mediating the synthesis of flavonoids in plants (Abe \& Morita, 480 2010).

481 Surprisingly, in the phylogenetic analysis comparing KS domains of FAS, PKS, and the putative 482 alkamide acyl chain biosynthetic genes, KS domains of the latter were found in a clade close to 483 PKS from microorganisms. Thus, PKS-related, specific genes, appear to be conserved within the 484 alkamide producer species, suggesting duplications that led to the evolution of novel functions. 485 A possibility is that these plants acquired these genes by horizontal transfer, as has been 486 suggested for other PKS gene families (Kohli et al., 2016) or alternative splicing that can give 487 rise to different transcript versions of a single gene. Previously, no KS domains resembling type 488 I PKS KS domains have been reported in plants, possibly because of initial low sequencing 489 depth. It would be very interesting to search carefully for similar transcripts in other plants, 490 especially those known to synthesize alkamides.

491 Because of the clustering of KS domains coded by upregulated transcripts with KS domains 492 found in type I PKS systems, found in certain microorganisms, we sought whether other similar 493 domains present in those systems were also found coded by other upregulated transcripts. These 494 results suggest alkamide synthesis is likely carried out by enzymes that are similar to type I PKS. 495 These findings are important, as the differentiation of PKS and FAS will facilitate approaches 496 investigating alkamides biosynthesis pathways in other plant species. They are also interesting as 497 this type of systems were previously thought to be absent from plant species, and mostly present 498 in microorganisms. One possibility is, of course, that these enzymes are expressed by endophytes 499 that are exclusively present in the root tissues of H. longipes. However, since RNA-Seq analysis 500 was performed using polyadenylated RNA, it is unlikely that prokaryotic RNA was sequenced, 501 though fungal transcripts would not have been filtered out. Nevertheless, we would expect fungal 502 transcripts to be present at much lower levels than plant transcripts. Moreover, putative 503 endophytes that are exclusively present in H. longipes roots have not been observed in the in 504 vitro root culture in the lab to date. 
505 To propose the novel alkamides biosynthetic pathway, we considered the chemical structure of 506 the affinin acyl chain, and the characteristics of the known products of FAS or PKS. Fatty acids 507 derived from FASs comprise completely reduced acyl chains, and the polyketides derived from 508 PKSs include partially unsaturated acyl chains. In the polyunsaturated fatty acids (PUFAs), the 509 double bonds are incorporated by fatty acid desaturases (FADs), iron-dependent 510 metalloenzymes, on the fatty acids released from the FAS enzymatic complex (Gagné et al., 511 2009; Shanklin et al., 2009). In contrast, the double bonds in the polyketides are the result of the 512 enzymatic activity of KR, DH, and ER domains (Hertweck, 2009) during the chain elongation.

513 The pathway proposes that the candidate enzymes are involved in the biosynthesis of the affinin 514 acyl chain in H. longipes roots through a pathway that is similar to the polyketide biosynthesis 515 pathway. This model is further referred as "PKS alk". The proposed PKS alk (Fig. 5), would 516 incorporate and keep double bonds during the elongation of the chain. In order to accomplish 517 this, it would contain four modules, which could be named: Aff-1, Aff-2, Aff-3, and Aff-4, 518 where each incorporates two carbons. It would require acyl-coenzyme A $(\mathrm{CoA})$ as the starter and 519 malonyl-acyl carrier protein (ACP) as the extender unit. The $\mathrm{C} 10-2 \mathrm{E}, 6 \mathrm{Z}, 8 \mathrm{E}$ as a polyunsaturated 520 acyl chain, would also require PKS alk to have a partial reduction activity to produce the 521 molecular structure of the acyl chain of alkamides. The selected set of genes contain the domains 522 required to support these reactions, although further studies are required to confirm their 523 participation in the synthesis of the acyl chain of alkamides. The proposed system (PKS alk) 524 would work in a modular fashion as in the known type I PKSs (Hertweck, 2009). This 525 multienzymatic mechanism is detailed in Figure 5. In order to obtain the affinin acyl chain 526 specific structure, the KR and DH domains would perform a partial reduction in modules 1 (Aff527 1), 2 (Aff-2), and 4 (Aff-4), which are referred to as $\gamma$-modules because they contain only KR 528 and DH domains. In comparison, a full keto group reduction would be provided by module 3 529 (Aff-3), which contains ER in addition to $\mathrm{KR}$ and $\mathrm{DH}$, and is referred to as the $\delta$-module in 530 agreement with the nomenclature recently suggested by Keatinge-Clay (2017). In this pathway, 531 the AT substrate would be malonyl-CoA, as observed in FASs and some other PKSs. After the 532 first two biosynthetic modules perform their activities, the conjugated double bonds in 4-trans 533 and 2-cis positions would be introduced, as the DH in the first module acts on a D- $\beta$-hydroxy534 acyl intermediate to form a trans double bond in the Aff- $1(\gamma)$ module. However, in the Aff-2 $(\gamma)$ 
535 module, the DH would generate a cis double bond during the dehydration of an L- $\beta$-hydroxy 536 intermediate. This mechanism, by means of which the $\mathrm{DH}$ generates cis double bonds when a 537 trans double bound is inserted in the previous module during the synthesis of an acyl chain, has 538 been observed in several PKS type I enzymes in microorganisms (Kalan et al., 2013). Once the 539 alkamide acyl chain is synthesized, the offloading machinery TE in the Aff-4 $(\gamma)$ module, would 540 be responsible for freeing the $\alpha$-unsaturated acyl chain from the PKS complex. This TE should 541 differ from the FASs TE. It would accept an $\alpha$ - unsaturated substrate and, by acting as a logic 542 gate, would determine the substrate's fate (Horsman et al., 2016). In the last step of the proposed 543 pathway, the TE would act specifically on the acyl $\alpha$-unsaturated chain, such as the affinin acyl 544 chain C10-2E,6Z,8E. Notably, this TE should be similar in all of the offloading alkamide PKSs 545 in order to maintain the characteristic $\alpha$-unsaturation of these structures. In addition, the TE may 546 be involved in the determination of the amine to which the acyl chain will be linked via an amide 547 bond, as the final step of alkamide biosynthesis. The TE of FASs and PKSs catalyze substrate 548 offloading from ACPs. Acyl chains bound to the arm of ACPs are loaded onto the active site 549 serine of a TE and then released via a nucleophilic attack (Horsman et al., 2016). Regarding 550 alkamide biosynthesis this enzyme should be specific, as it would transfer the chain to an amide 551 and not to an ester, and should support the obligated presence of an $\alpha$-unsaturated acyl chain as 552 defined for alkamides. More detailed studies would be required to explain the presence of the 553 bornyl ester of C10-2E,6Z,8E reported for H. longipes roots only (Molina-Torres et al., 1995).

554 The "PKS alk" model can be used as a basis to design experiments to demonstrate it is indeed 555 responsible for alkamide biosynthesis, and to better understand it. According to this mechanism, there would be a great similarity between PKS alk and plastid type I FAS biosynthetic pathway.

557 Both are giant multienzymes complexes catalyzing all steps of the biosynthesis from acetyl- and 558 malonyl-CoA precursor extension and utilizing the $\mathrm{KR}, \mathrm{DH}$, and $\mathrm{ER}$, to reduce the keto group.

559 However, the main difference between the PKS alk and Type I FAS would be that the FAS type 560 I is constituted by seven modules, while the proposed PKS alk, contains four putative modules. 561 In modules one, two and four of the PKS alk the enzymatic domain with ER activity would be 562 absent, while in FAS, all modules are similar and contain the ER domain. Besides, while the 563 FAS is present in all plants, the alkamides have been reported only in specific plant species of 564 different genus and families, suggesting that the PKS alk also presents a limited distribution 
565 among the plant species. Although several plant species of taxonomically different families

566 produce alkamides such as the Asteraceae, Piperaceae, and Rutaceae, the PKS alk pathway, 567 proposed in this study, maybe also mediating the biosynthesis of all the alkamides in those 568 species phylogenetically separated e.g. Zhantoxylum spp (Rutaceae) and Ctenium aromaticum 569 (Poaceae).

\section{Conclusions}

571 In the present study, the first transcriptomic high-throughput sequencing of the Mexican endemic 572 plants $H$. longipes and $H$. аnпиa is described. The comparative transcriptome analysis performed 573 for the two Heliopsis species led to the selection of a set of candidate genes putatively involved 574 in the affinin acyl chain biosynthesis, allowing to propose a model for alkamide acyl chain 575 biosynthesis by a PKS complex, here named PKS alk. This complex would be partially reductive 576 and related to type I PKSs, and present in plants that produce alkamides. These findings provide

577 a framework for molecular and biochemical studies to confirm the activity of the proposed 578 pathway. Further studies can also help to better understand the underlying regulatory 579 mechanisms involved and the physiological role of these multifunctional molecules in plants.

\section{Acknowledgements}

581 We thank Enrique Ibarra-Laclette, Juan Carlos Ochoa Sánchez for their technical support in this 582 project, David Ramírez-Noya for the collection and taxonomic identification of the Heliopsis species, Elias Nieto Resendiz for providing Heliopsis longipes tissues, and Laila PartidaMartinez, Francisco Barona Gómez, Luis José Delaye, and Carolyne Smith, a Peace Corps Response volunteer, for reading the manuscript.

\section{Abbreviations}

587 ACP: acyl carrier protein; AT: acyl transferase; DH: dehydratase; ER: enoyl reductase; FAS:

588

589

590 fatty acids synthetase; GO: gene ontology; KR: keto reductase; KS; ketosynthase; OR: oxide reductase; PKS: Polyketide synthase; RNA-Seq: RNA sequencing; RT-qPCR: quantitative real time polymer chain reaction; TE: thioesterase; TF: transcription factor. 


\section{References}

593

594

595

596

597

598

599

600

601

602

603

604

605

606

607

608

609

610

611

612

613

614

615

616

617

618

619

620

621

622

623

624

625

626

627

628

629

630

631

Abe I, and Morita H. 2010. Structure and function of the chalcone synthase superfamily of plant type III polyketide synthases. Natural Products Reports 27:809-838. 10.1039/B909988N

Ambawat S, Sharma P, Yadav NR, and Yadav RC. 2013. MYB transcription factor genes as regulators for plant responses: an overview. Physiology and Molecular Biology of Plants 19:307-321. 10.1007/s12298-013-0179-1

Ashburner M, Ball CA, Blake JA, Botstein D, Butler H, Cherry JM, Davis AP, Dolinski K, Dwight SS, Eppig JT, Harris MA, Hill DP, Issel-Tarver L, Kasarskis A, Lewis S, Matese JC, Richardson JE, Ringwald M, Rubin GM, and Sherlock G. 2000. Gene Ontology: tool for the unification of biology. Nature Genetics 25:25. 10.1038/75556

Barbosa AF, Silva KCB, de Oliveira MCC, de Carvalho MG, and Sabaa Srur AUO. 2016. Effects of Acmella oleracea methanolic extract and fractions on the tyrosinase enzyme. Revista Brasileira de Farmacognosia. http://dx.doi.org/10.1016/j.bjp.2016.01.004

Bolger AM, Lohse M, and Usadel B. 2014. Trimmomatic: a flexible trimmer for Illumina sequence data. Bioinformatics 30:2114-2120. 10.1093/bioinformatics/btu170

Boonen J, Bronselaer A, Nielandt J, Veryser L, De Tré G, and De Spiegeleer B. 2012. Alkamid database: Chemistry, occurrence and functionality of plant N-alkylamides. Journal of Ethnopharmacology 142:563-590. 10.1016/j.jep.2012.05.038

Brown AP, Slabas AR, and Rafferty JB. 2009. Fatty Acid Biosynthesis in Plants - Metabolic Pathways, Structure and Organization. In: Wada H, and Murata N, eds. Lipids in Photosynthesis: Essential and Regulatory Functions. Dordrecht: Springer Science + Business Media B.V., 11-34.

Bustin, S. A., Benes, V., Garson, J. A., Hellemans, J., Huggett, J., Kubista, M., ... \& Vandesompele, J. (2009). The MIQE Guidelines: Minimum Information for Publication of Q uantitative Real-Time PCR Experiments.

Chenna R. 2003. Multiple sequence alignment with the Clustal series of programs. Nucleic Acids Research 31:3497-3500. 10.1093/nar/gkg500

Christensen LP, and Lam J. 1991. Acetylenes and related compounds in Heliantheae. Phytochemistry 30:11-49.

Cortez-Espinosa N, Aviña-Verduzco JA, Ramírez-Chávez E, Molina-Torres J, and Rios-Chávez P. 2011. Valine and Phenylalanine as Precursors in the Biosynthesis of Alkamides in Acmella radicans. Natural Product Communications 6 857-861.

Drobak BK, Franklin-Tong VE, and Staiger CJ. 2004. The role of the actin cytoskeleton in plant cell signaling. New Phytologist 163:13-30. 10.1111/j.1469-8137.2004.01076.x

Fu L, Niu B, Zhu Z, Wu S, and Li W. 2012. CD-HIT: accelerated for clustering the nextgeneration sequencing data. Bioinformatics 28:3150-3152. 10.1093/bioinformatics/bts565

Gagné SJ, Reed DW, Gray GR, and Covello PS. 2009. Structural Control of Chemoselectivity, Stereoselectivity, and Substrate Specificity in Membrane-Bound Fatty Acid Acetylenases and Desaturases. Biochemistry 48:12298-12304. 10.1021/bi901605d

Peer] reviewing PDF | (2020:01:45349:2:0:NEW 4 Aug 2020) 
632 Gallo A, Ferrara M, and Perrone G. 2013. Phylogenetic study of polyketide synthases and 633 nonribosomal peptide synthetases involved in the biosynthesis of mycotoxins. Toxins

634 (Basel) 5:717-742. 10.3390/toxins5040717

635

636

637

638

639

640

641

642

643

644

645

646

647

648

649

650

651

652

653

654

655

656

657

658

659

660

661

662

663

664

665

666

667

668

669

670

Garber M, Grabherr MG, Guttman M, and Trapnell C. 2011. Computational methods for transcriptome annotation and quantification using RNA-seq. Nature Methods 8:469-477. 10.1038/nmeth.1613

García-Chávez A, Ramírez-Chávez E, and Molina-Torres J. 2004. El Género Heliopsis (Heliantheae; Asteraceae) en México y las alcamidas presentes en sus raíces. Acta Botánica Mexicana 69:115-131.

Greger H. 1984. Alkamides: Structural Relationships, Distribution and Biological Activity. Planta Medica 50:366-375.

Greger H. 2015. Alkamides: a critical reconsideration of a multifunctional class of unsaturated fatty acid amides. Phytochemistry Reviews:1-42. 10.1007/s11101-015-9418-0

Guo A, He K, Liu D, Bai S, Gu X, Wei L, and Luo J. 2005. DATF: a database of Arabidopsis transcription factors. Bioinformatics 21:2568-2569. 10.1093/bioinformatics/bti334

Gupta RS, Naushad S, Chokshi C, Griffiths E, and Adeolu M. 2015. A phylogenomic and molecular markers based analysis of the phylum Chlamydiae: proposal to divide the class Chlamydiia into two orders, Chlamydiales and Parachlamydiales ord. nov., and emended description of the class Chlamydiia. Antonie Van Leeuwenhoek 108:765-781. 10.1007/s10482-015-0532-1

Hertweck C. 2009. The Biosynthetic Logic of Polyketide Diversity. Angewandte Chemie International Edition 48:4688-4716. 10.1002/anie.200806121

Horsman ME, Hari TPA, and Boddy CN. 2016. Polyketide synthase and non-ribosomal peptide synthetase thioesterase selectivity: logic gate or a victim of fate? Natural Products Reports 33:183-202. 10.1039/C4NP00148F

Jones DT, Taylor WR, and Thornton JM. 1992. The rapid generation of mutation data matrices from protein sequences. Bioinformatics 8:275-282. 10.1093/bioinformatics/8.3.275

Kalan L, Gessner A, Thaker Maulik N, Waglechner N, Zhu X, Szawiola A, Bechthold A, Wright Gerard D, and Zechel David L. 2013. A Cryptic Polyene Biosynthetic Gene Cluster in Streptomyces calvus Is Expressed upon Complementation with a Functional bldA Gene. Cell Chemical Biology 20:1214-1224. 10.1016/j.chembiol.2013.09.006

Kamitani M, Nagano AJ, Honjo MN, and Kudoh H. 2016. RNA-Seq reveals virus-virus and virus-plant interactions in nature. FEMS Microbiology Ecology 92. $10.1093 / \mathrm{femsec} / \mathrm{fiw} 176$

Keatinge-Clay AT. 2017. Polyketide Synthase Modules Redefined. Angewandte Chemie International Edition 56:4658-4660. 10.1002/anie.201701281

Kohli GS, John U, Van Dolah FM, and Murray SA. 2016. Evolutionary distinctiveness of fatty acid and polyketide synthesis in eukaryotes. The ISME journal 10:1877-1890. 10.1038/ismej.2015.263

Peer) reviewing PDF | (2020:01:45349:2:0:NEW 4 Aug 2020) 
671 Kumar S, Stecher G, and Tamura K. 2016. MEGA7: Molecular Evolutionary Genetics Analysis

672 Version 7.0 for Bigger Datasets. Molecular Biology and Evolution 33:1870-1874.

$673 \quad 10.1093 / \mathrm{molbev} / \mathrm{msw} 054$

674

Langmead B, Trapnell C, Pop M, and Salzberg SL. 2009. Ultrafast and memory-efficient

675

676 alignment of short DNA sequences to the human genome. Genome Biology 10:R25-R25. $10.1186 / \mathrm{gb}-2009-10-3-\mathrm{r} 25$

677

678

679

680

681

682

683

684

685

686

687

688

689

690

691

692

693

694

695

696

697

698

699

700

701

702

703

704

705

706

707

708

709

710

Li B, and Dewey CN. 2011. RSEM: accurate transcript quantification from RNA-Seq data with or without a reference genome. BMC Bioinformatics 12:323. 10.1186/1471-2105-12-323

Livak KJ, and Schmittgen TD. 2001. Analysis of relative gene expression data using real-time quantitative PCR and the 2(-Delta Delta C(T)) Method. Methods 25:402-408. 10.1006/meth.2001.1262

Medema MH, and Osbourn A. 2016. Computational genomic identification and functional reconstitution of plant natural product biosynthetic pathways. Natural Product Reports 33:951-962. 10.1039/C6NP00035E

Méndez-Bravo A, Calderon-Vázquez C, Ibarra-Laclette E, Raya-Gonzalez J, Ramírez-Chávez E, Molina-Torres J, Guevara-García AA, López-Bucio J, and Herrera-Estrella L. 2011. Alkamides Activate Jasmonic Acid Biosynthesis and Signaling Pathways and Confers Resistance to Botrytis cinerea in Arabidopsis thaliana. Plos ONE 6:e27251.

Minto RE, and Blacklock BJ. 2008. Biosynthesis and function of polyacetylenes and allied natural products Progress in Lipid Research 47:233-306. S0163-7827(08)00015-5 [pii]

10.1016/j.plipres.2008.02.002

Molina-Torres J, Salgado-Garciglia R, Ramirez-Chavez E, and del-Rio RM. 1995. Presence of the bornyl ester of deca-2E,6Z,8E-trienoic acid in Heliopsis longipes roots. J Nat Prod (Lloydia) 58:1590-1591.

Osbourn AE, and Lanzotti V. 2009. Plant-derived Natural Products. Synthesis, Function, and Application. Springer Science+Business Media.

Parmar VS, Jain SC, Bisht KS, Jain R, Taneja P, Jha A, Tyagi OD, Prasad AK, Wengel J, Olsen CE, and Bool PM. 1997. Phytochemistry of the genus Piper. Phytochemistry 46:597-673.

Piel J. 2009. Metabolites from symbiotic bacteria. Nat Prod Rep 26:338-362. 10.1039/b703499g

Piel J. 2010. Biosynthesis of polyketides by trans-AT polyketide synthases. Nat Prod Rep 27:996-1047. 10.1039/b816430b

Ramírez-Chávez E, López-Bucio J, Herrera-Estrella L, and Molina-Torres J. 2004. Alkamides Isolated from Plants Promote Growth and Alter Root Development in Arabidopsis. Plant Physiol 134:1058-1068.

Ramírez-Noya D, González-Elizondo MS, and Molina-Torres J. 2011. Heliopsis suffruticosa (Compositae, Heliantheae), una nueva especie del occidente de Zacatecas. Acta Botánica Mexicana 97:39-47.

Rizhsky L, Jin H, Shepard MR, Scott HW, Teitgen AM, Perera MA, Mhaske V, Jose A, Zheng X, Crispin M, Wurtele ES, Jones D, Hur M, Góngora-Castillo E, Buell CR, Minto RE, and Nikolau BJ. 2016. Integrating metabolomics and transcriptomics data to discover a

Peer] reviewing PDF | (2020:01:45349:2:0:NEW 4 Aug 2020) 
711

712

713

714

715

716

717

718

719

720

721

722

723

724

725

726

727

728

729

730

731

732

733

734

735

736

737

738

739

740

741

742

743

744

biocatalyst that can generate the amine precursors for alkamide biosynthesis. The Plant Journal 88:775-793. 10.1111/tpj.13295

Robinson MD, McCarthy DJ, and Smyth GK. 2010. edgeR: a Bioconductor package for differential expression analysis of digital gene expression data. Bioinformatics 26:139140. 10.1093/bioinformatics/btp616

Shanklin J, Guy JE, Mishra G, and Lindqvist Y. 2009. Desaturases: emerging models for understanding functional diversification of diiron-containing enzymes. J Biol Chem 284:18559-18563. 10.1074/jbc.R900009200

Surup F, Viehrig K, Mohr KI, Herrmann J, Jansen R, and Muller R. 2014. Disciformycins A and B: 12-membered macrolide glycoside antibiotics from the myxobacterium Pyxidicoccus fallax active against multiresistant staphylococci. Angewandte Chemie International Edition 53:13588-13591. 10.1002/anie.201406973

Xiao M, Zhang Y, Chen X, Lee E-J, Barber CJS, Chakrabarty R, Desgagné-Penix I, Haslam TM, Kim Y-B, Liu E, MacNevin G, Masada-Atsumi S, Reed DW, Stout JM, Zerbe P, Zhang Y, Bohlmann J, Covello PS, De Luca V, Page JE, Ro D-K, Martin VJJ, Facchini PJ, and Sensen CW. 2013. Transcriptome analysis based on next-generation sequencing of nonmodel plants producing specialized metabolites of biotechnological interest. Journal of Biotechnology 166:122-134. https://doi.org/10.1016/j.jbiotec.2013.04.004

Yang X. 2008. Aroma Constituents and Alkylamides of Red and Green Huajiao (Zanthoxylum bungeanum and Zanthoxylum schinifolium). Journal of Agricultural and Food Chemistry 56:1689-1696. 10.1021/jf0728101

You FM, Li P, Kumar S, Ragupathy R, Li Z, Fu Y-B, and Cloutier S. 2014. Genome-wide Identification and Characterization of the Gene Families Controlling Fatty Acid Biosynthesis in Flax (Linum usitatissimum L). Journal of Proteomics \& Bioinformatics 07. 10.4172/jpb.1000334

Wang, Y. N., Tang, L., Hou, Y., Wang, P., Yang, H., \& Wei, C. L. (2016). Differential transcriptome analysis of leaves of tea plant (Camellia sinensis) provides comprehensive insights into the defense responses to Ectropis oblique attack using RNA-Seq. Functional \& integrative genomics, 16(4), 383-398.

Zhang, N., \& Deyholos, M. K. (2016). RNASeq analysis of the shoot apex of flax (Linum usitatissimum) to identify phloem fiber specification genes. Frontiers in plant science, 7 , 950. 


\section{Figure 1}

General structure of alkamides (alk) and the main alkamides produced in Heliopsis longipes roots.

A) R1: $\alpha$-unsaturated acyl chain C8 to C18. Other double bonds may be present isolated $(E)$ or conjugated $(E, E$ or $E, Z)$. R2: amine. R3: mostly $\mathrm{H}$. B) Structure of affinin (Nisobutyl-2E,6Z,8E-decatrienamide) the most abundant, represents over $90 \%$ of the total alkamides in roots. Minor alkamides are: C) N-2-methylbutyl-2E,6Z,8E-decatrienamide (3.3\%), D) $N$-isobutyl-2E-en-8,10-diyn-undecanamide (2.5\%), and E) decanoic-2E,6Z,8Ebornyl-ester (1.1\%). The latter, although not an alkamide, presents the same acyl moiety as the denoted alkamides.

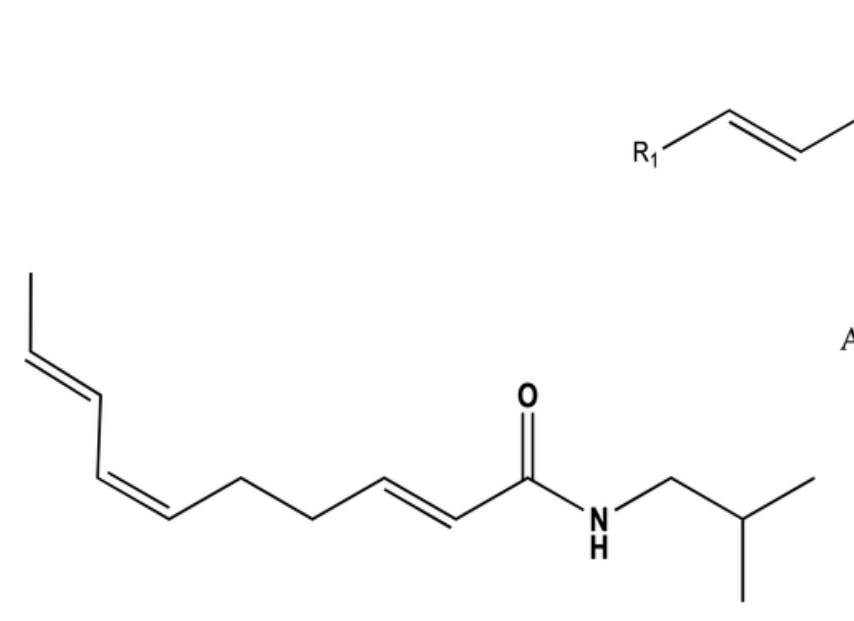

B

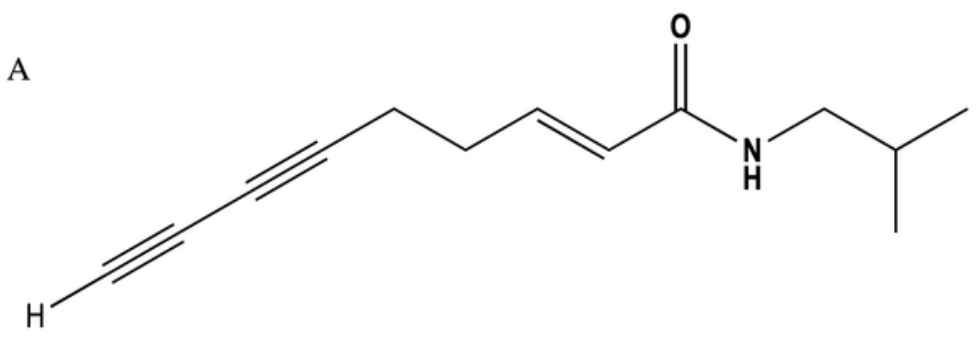

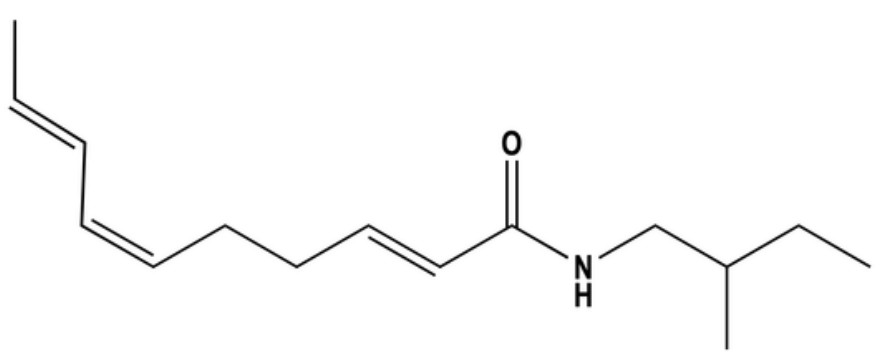

D

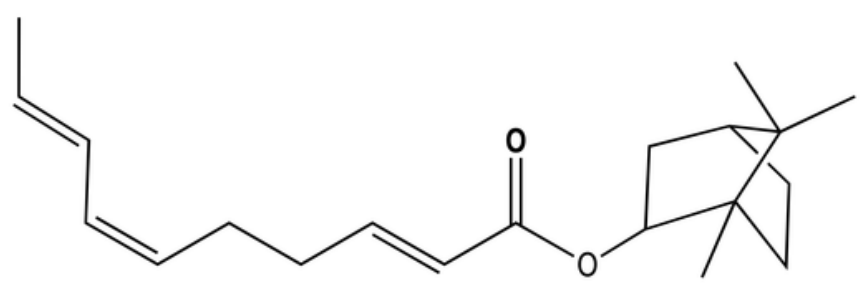

E 
Figure 2

Gene Ontology (GO) classification of unigenes derived from RNA-Seq in Heliopsis species studied.

A total of 29,316 unigenes in $H$. longipes and 29,870 unigenes in $H$. annua were successfully annotated and classified into three GO categories: Cellular Component, Biological Process and Molecular Function. 
Transcripts Abundance

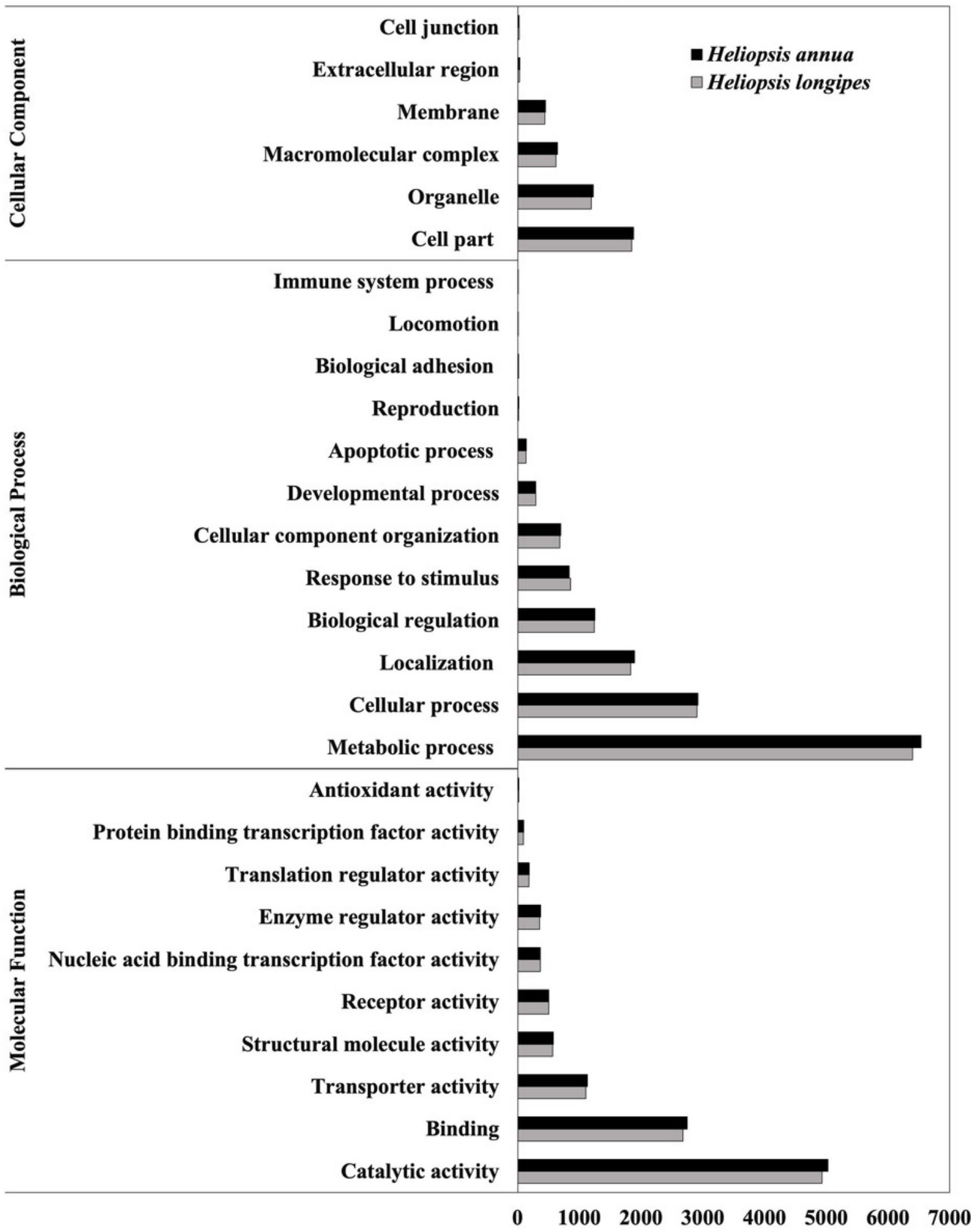




\section{Figure 3}

Relative expression of candidate genes involved in the putative acyl-chain biosynthetic pathway identified by differential gene expression analysis of Heliopsis longipes and Heliopsis annua roots and leaves transcriptomes.

A) Quantitative real-time PCR. B) Expression, in fragments per kilobase of exon model per million mapped reads (FPKM) from the RNA sequencing data. ACP: acyl carrier protein, AT: acyl transferase, DH: dehydratase, KS: ketoacyl synthase, OR: oxide reductase including the $\mathbf{K}$ : keto reductase and ER: enoyl reductase; DH: dehydratase, and TE: thioesterase. The number corresponds to the transcript set identified by differential expression analysis.Error bars represent standard error for $n=2$. The relative expression pattern of the candidate transcripts in the non-alkamide producing tissue was close to zero and therefore is not easily observed in the graphs. 
A)

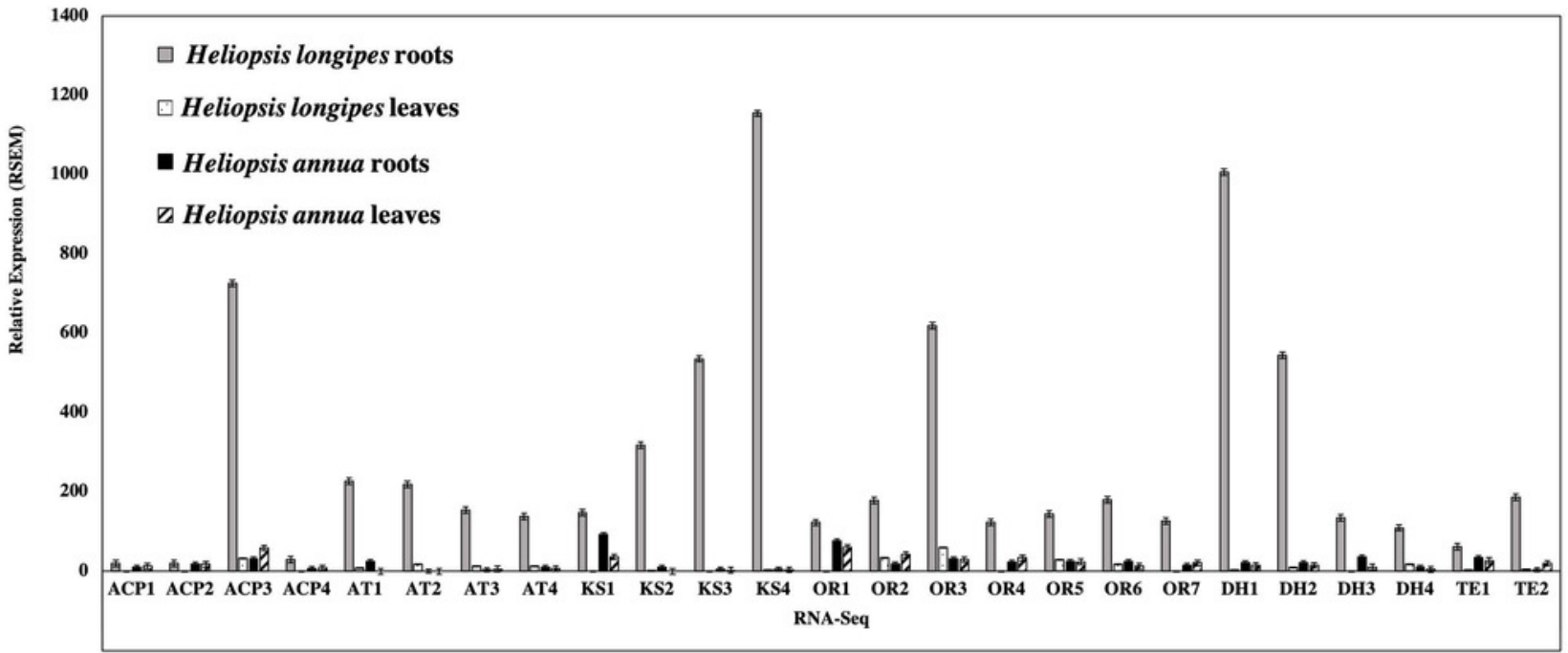

B)

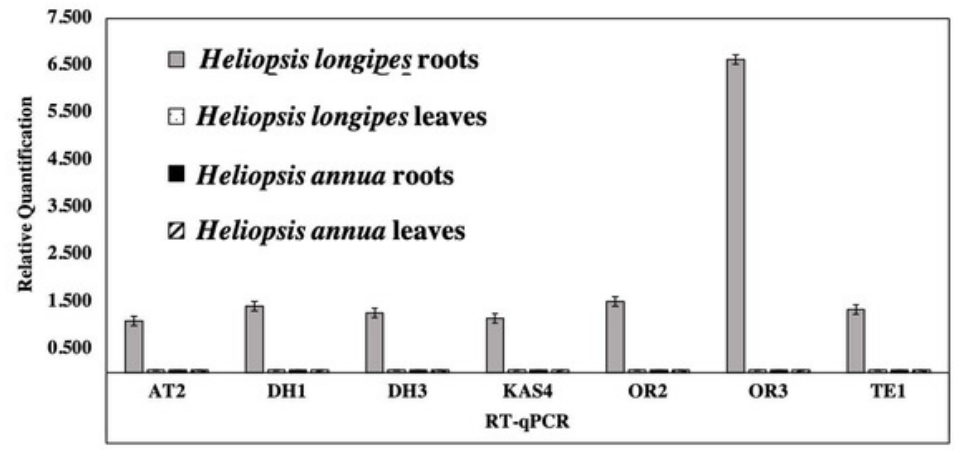




\section{Figure 4}

Molecular phylogenetic analysis of the ketoacyl synthase (KS) domains protein sequences.

KS domain protein sequences differentially expressed in $H$. longipes and $H$. annua roots and leaves tissue were selected for phylogenetic analysis. KS domain protein sequences nondifferentially expressed but annotated in the transcriptomes that coded for conserved domains of fatty acid synthases (FAS) and polyketide synthase (PKS) were used as comparison. The different $K S$ domains selected were classified as group $A, C, D, E$ or $F$ for non-differentially expressed transcripts from $H$. annua (roots and leaves) and $H$. longipes (leaves). The group B for the upregulated transcripts in $H$. longipesroots. KS domains from: Echinacea purpura from Plant Biology Department, Michigan State University; and other KS domains of FAS and PKS from mammals, microorganisms and other plants, obtained from $\mathrm{NCBI}$, were also were selected for phylogenetic analysis based on the Maximum Likelihood method. The numbers at nodes indicate bootstrap support, for 100 bootstrap replications. The analysis was conducted in MEGA 7. 


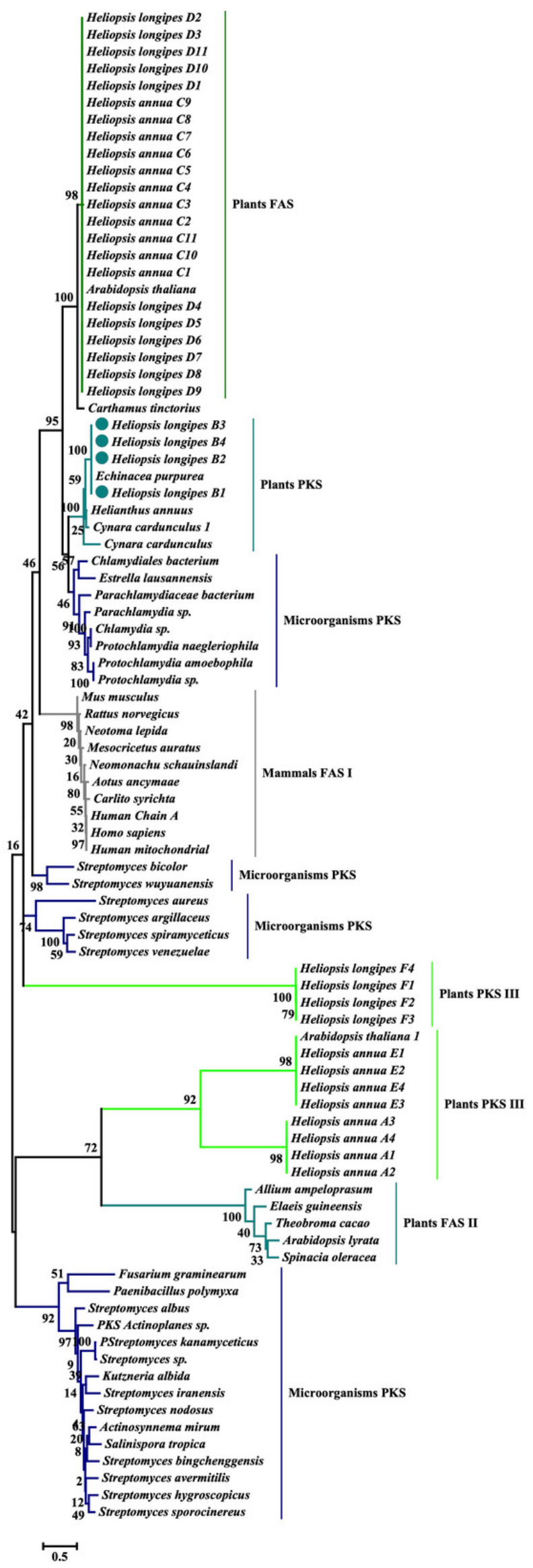

Peer] reviewing PDF | (2020:01:45349:2:0:NEW 4 Aug 2020) 


\section{Figure 5}

Proposed affinin acyl chain biosynthesis mediated by polyketide synthase (PKS).

The proposed biosynthetic pathway exhibits a multi-modular architecture and requires acylCoA as the starter unit and malonyl-ACP as the extender unit. The KS domain catalyzes a decarboxylative Claisen $\left(\mathrm{CO}_{2}\right)$ condensation between the growing chain and an extender unit attached to the ACP domain (gray circles), whereas the $\mathbf{A T}$ domain selects and loads an extender unit to the ACP. The oxide-reductase that includes the $\mathbf{K R}$ and $\mathbf{D H}$ domains sequentially reduces the $\beta$-keto group to a $\beta$-hydroxy group and $\alpha$-double bond. Additional ER domains reduce the $\alpha$-double bond to a saturated product, and TE cleaves the synthesized acyl chain. Enzymatic domains: ACP: acyl carrier protein, AT: acyl transferase, KS: ketoacyl synthase, KR: keto reductase, DH: dehydratase, ER: enoyl reductase, TE: thioesterase.

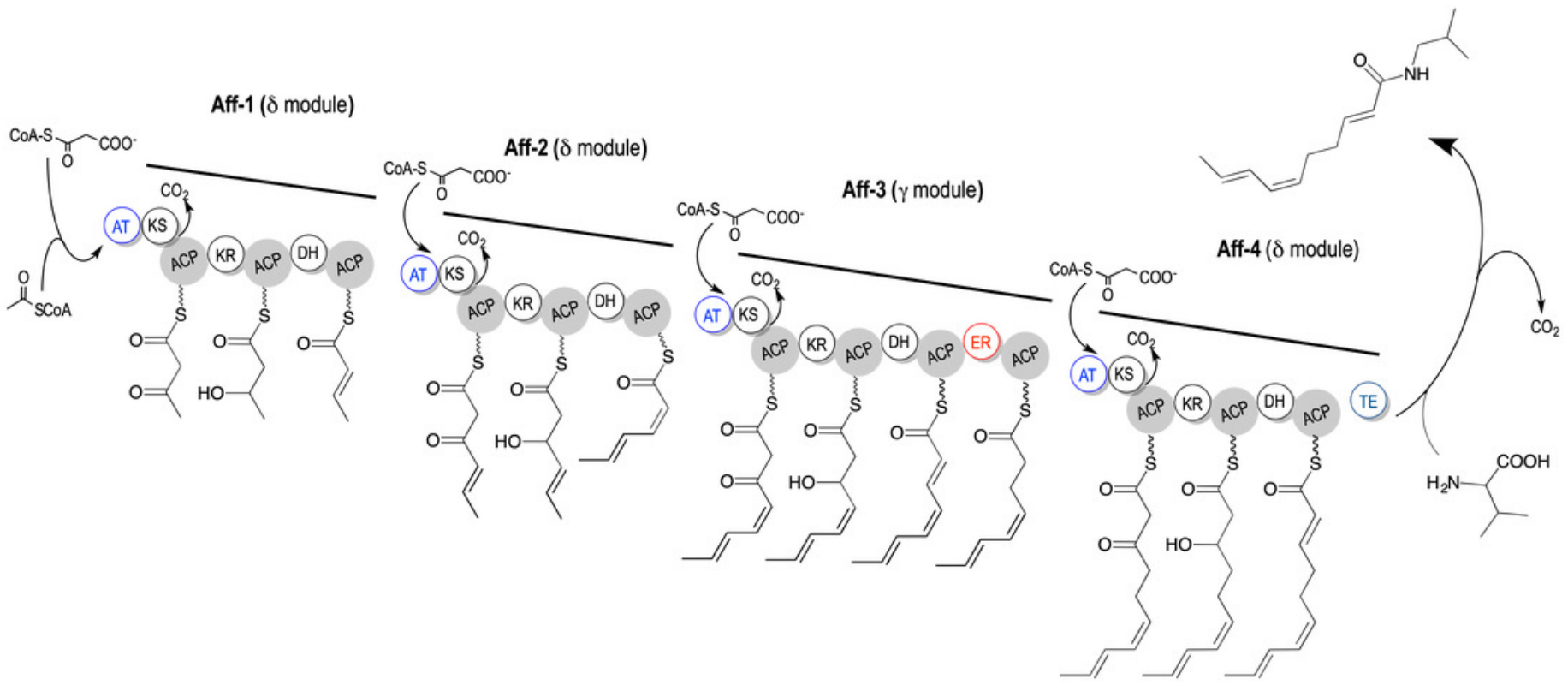




\section{Table $\mathbf{1}$ (on next page)}

RNA sequencing (RNA-Seq) and de novo assembly data for the roots and leaves transcriptomes. 
1 Table 1. RNA sequencing (RNA-Seq) and de novo assembly data for the roots and leaves 2 transcriptomes.

\begin{tabular}{lccccc}
\hline A. RNA-Seq & \multicolumn{1}{c}{ Total } \\
\hline Sample & $\begin{array}{c}\text { cDNA } \\
\text { library (bp) }\end{array}$ & Read 1 & Read 2 & Run mode & (GB) \\
\hline Heliopsis longipes roots 1 & 417 & $22,249,016$ & $22,249,016$ & \\
Heliopsis longipes roots 2 & 854 & $23,373,302$ & $23,373,302$ & \\
Heliopsis longipes leaves 1 & 339 & $16,065,446$ & $16,065,446$ & \\
Heliopsis longipes leaves 2 & 697 & $15,666,029$ & $15,666,029$ & $2 \times 100$ bp & 27.7 \\
Heliopsis annua roots 1 & 448 & $15,518,097$ & $15,518,097$ & \\
Heliopsis annua roots 2 & 474 & $14,808,022$ & $14,808,022$ & \\
Heliopsis annua leaves 1 & 497 & $15,754,789$ & $15,754,789$ & & \\
Heliopsis annua leaves 2 & 483 & $14,334,639$ & $14,334,639$ &
\end{tabular}

B. de novo assembly

Heliopsis longipes

$$
150,124,843
$$$$
165,770
$$$$
14,461
$$

906

200
Heliopsis annua

$115,219,511$

155,457

15,574

695

200

3 